\title{
Uma abordagem dinamica da questão da competencia, conhecimento pessoal e conhecimento academico
}

\author{
A dynamic approach to the issue of competence, \\ personal knowledge and academic knowledge
}

\section{Un enfoque dinámico de la cuestión de la competencia, del conocimiento personal y del conocimiento académico.}

\section{Une approche dynamique de la competence, des savoirs et de la connaissance}

RESUMO: Adotamos uma perspectiva antropológica sobre trabalho e aprendizagem, que é também a perspectiva da ergologia. Isto permite-nos ver a competência de uma forma dinâmica.A representação dinâmica opõe-se a uma representação estática da competência. A representação estática é denunciada em todos os lugares como desumanizante, pois só considera o ato profissional de forma impessoal. A pessoa humana é supostamente intercambiável e sua atividade é ignorada. Pelo contrário, como vemos no diagrama dos "seis ingredientes da competência", a abordagem ergológica quer manter unido o ato e o ator. Não devem ser separados, mas também não devem ser confundidos. É a representação dinâmica da competência que permite: (a) não violentar à atividade humana, reconhecendo o sujeito em seu ato quando está em situação de trabalho; (b) e, no entanto, ser capaz de distinguir o ato profissional de forma impessoal, de modo a permitir a organização do trabalho e da formação - e a produção de quadros de referência.

Palavras-chave:Competência.Atividade humana.Situação de trabalho.Padrões de formação.

ABSTRACT: We adopt an anthropological perspective on work and learning, which is also the perspective of ergology. This allows us to look at the competence in a dynamic way. Dynamic representation is opposed to a static representation of competence. A static representation is 1 Université de Strasbourg, Espe, Laboratoire LISEC, France. eMail : louis.durrive@unistra.fr

ISSN 1982-8829 Tempus, actas de saúde colet, Brasília, 13(2), 217-233, jun, 2019. 
everywhere denounced as dehumanizing because it only considers the professional act in an impersonal way. The human person is supposedly interchangeable and his activity is ignored. On the contrary, as we see with the diagram of the "six ingredients of competence", the ergological approach wants to hold together the act and the actor. They should not be separated, but neither should they be confused. It is the dynamic representation of the competence that allows: (a) no longer to do violence to human activity by recognizing the subject in his act when he is in a work situation; (b) and nevertheless to be able to distinguish the professional act in an impersonal way, so as to allow the organization of work and training and the production of reference frames.

Keywords:Competence. Human activity. Work situation. Training standards.

RESUMEN: Adoptamos una perspectiva antropológica sobre el trabajo y el aprendizaje, que es también la perspectiva de la ergología. Esto nos permite ver la competencia de una manera dinámica. La representación dinámica se opone a una representación estática de la competencia. La representación estática es denunciada en todas partes como deshumanizadora porque sólo considera el acto profesional de manera impersonal. La persona humana es supuestamente intercambiable y su actividad es ignorada. Por lo contrario, como vemos en el diagrama de los "seis ingredientes de la competencia", el enfoque ergológico quiere mantener unidos el acto y el actor. No deben separarse, pero tampoco deben confundirse. Es la representación dinámica de la competencia lo que permite: a) dejar de violentar la actividad humana al reconocer al sujeto en su acto cuando se encuentre en una situación de trabajo; b) y, sin embargo, poder distinguir el acto profesional desde un punto de vista impersonal, a fin de permitir la organización del trabajo y de la formación, así como la elaboración de marcos de referencia.

Palabras clave:Competencia actividad humana. Situación laboral. Estándares de formación.

RÉSUMÉ:A partir d'un éclairage anthropologique sur le travail et l'apprentissage, celui de l'ergologie, nous pouvons regarder la compétence dans une perspective dynamique. Celleci s'oppose à une représentation statique de la compétence, qui est partout dénoncée comme déshumanisante dans la mesure où l'acte professionnel est le seul à être pris en compte, de façon impersonnelle. La personne humaine est regardée comme interchangeable et son activité est ignorée.Au contraire, comme l'illustre le schéma des six ingrédients de la compétence, la démarche ergologique veut tenir ensemble l'acte et l'acteur. Il s'agit de ne pas les séparer mais aussi de ne pas les confondre. C'est la représentation dynamique de la compétence qui permet : (a) de ne plus faire violence à l'activité humaine en reconnaissant le sujet dans son acte lorsqu'il est en situation de travail ; (b) de pouvoir tout de même considérer l'acte professionnel en tant que tel, donc sous un angle impersonnel, de façon à permettre l'organisation du travail et de la formation et l'établissement des référentiels.

Mots-clés: Compétence.Activité humaine. Situation de travail. Référentiels de formation. 


\section{INTRODUCTION}

Dans un monde où l'anticipation est devenue un enjeu majeur, une approche par les connaissances ne semble plus suffire. Qu'elles soient orientées vers le travail ou vers la formation, les organisations choisissent de plus en plusd'adopter une approche par les compétences. Celle-ci veut répondre au double impératif de comprendre le monde et de le transformer. Il s'agit de développer partout où c'est possible des apprentissages qui favorisent autant la maîtrise des savoirs que le passage à l'action.

Ces évolutions constatées à l'échelle planétaire correspondent sans doute à une ère nouvelle pour l'humanité, celle qui a été ouverte par la globalisation et l'essor de la société cognitive. Cependant la déclinaison de ces ambitions fait surgir dans la pratique un vocabulaire très diversifié et parfois confus, avec des interprétations le plus souvent en tension. On croise les savoirs (savoirs savants, savoirs d'expérience), les connaissances (scientifiques, personnelles), les compétences (techniques, transversales), les savoir-faire et les savoir-être, les potentiels et les talents, etc.

Nous proposons ici une réflexion qui tente de réinscrire ces multiples notions dans une perspective anthropologique, celle de Georges Canguilhem, qui est au fondement de la démarche ergologique d'Yves Schwartz(1). Nous verrons qu'en évoquant le savoir, la connaissance ou la compétence, nous parlons en réalité de l'être humain. Si nous oublions cette référence, les mots sonnent creux car chacun de ces termes cherche à dire quelque chose du rapport de l'humain au monde et à son milieu de vie en particulier.

\section{VIVRE, C'EST FAIRE ARRIVER QUELQUE CHOSE}

L'expérience du monde pour chacun de nous depuis notre naissance, c'est l'expérience des autres et progressivement l'expérience du monde au sens large : le monde auquel nous nous heurtons, celui qui nous résiste. En tant que débutant dans l'aventure humaine, chacun va tirer des leçons de ce qui lui arrive. Nous tentons de faire quelque chose, ça nous résiste. Nous n'abandonnons pas, nous ne nous laissons pas faire, ce qui veut dire qu'à notre tour nous résistons et nous en tirons une leçon pour la prochaine fois.

Cela commence avec les jouets dans le berceau, puis à la crèche, au jardin d'enfants, à l'école, dans la vie quotidienne et bien plus tard : au travail, dans la vie active. Vivre, c'est échanger en continu et en permanence avec le monde matériel et humain qui nous entoure, avec les milieux que nous avons rencontrés, des milieux pluriels que nous ne cessons de traverser dans tous les sens des milieux sociaux, des milieux qui nous contraignent, qui cherchent à nous soumettre, mais aussi des milieux dans lesquels nous cherchons à vivre singulièrement, avec notre manière de voir, avec nos partis pris. Nous tentons de reprendre l'initiative sans forcément y parvenir, mais au moins en essayant d'être un peu plus actifs que passifs.

Pour chacune et chacun d'entre nous, vivre c'est dialoguer avec le monde qui est déjà là au ISSN 1982-8829 Tempus, actas de saúde colet, Brasília, 13(2), 217-233, jun, 2019. 
moment où nous arrivons ; dialoguer pour y faire quelque chose, mais aussi d'épreuve en épreuve, pour grandir soi-même, chercher à se construire à travers l'expérience d'un univers dans lequel nous n'allons pas nous contenter de subir. Car tout ce qui nous arrive ne relève pas seulement de contraintes qui nous font plier, qui nous assujettissent. Il y a un certain nombre de choses contraignantes que nous parvenons à tourner à notre avantage, sur lesquelles nous pouvons nous appuyer pour avancer. Vivre en effet correspond pour nousà une double exigence : celle de prendre en compte ce qui nous arrive, mais aussi celle de tenter de faire arriver quelque chose, d'être auteur d'une transformation (si modeste soit-elle) et producteur de sens. SelonCanguilhem(2),c'est une exigence de santé :

« Je me porte bien dans la mesure où je me sens capable de porter la responsabilité de mes actes, de porter des choses à l'existence et de créer entre les choses des rapports qui ne leur viendraient pas sans moi, mais qui ne seraient pas ce qu'ils sont sans elles ».

Songeons à n'importe quelle séquence de notre aventure humaine : le sportif qui prend appui sur la roche pour grimper; le romancier qui prend appui sur la structure de la langue pour rêver et faire rêver ; l'ingénieur qui prend appui sur les lois physiques pour édifier un pont, etc. La liste est sans fin - d'autant plus qu'elle commence dans les actes les plus ordinaires du quotidien.

Il est bon de prendre conscience de cette conquête sans cesse renouvelée de nos marges de manœuvre, de cette quête que chacun de nous mène inlassablement afin de comprendre ce qui l'entoure : pas forcément avec l'intention de satisfaire sa curiosité, mais parce que pour un être humain, c'est une condition pour vivre. Il nous est impossible d'agir, de faire quelque chose, d'intervenir dans le monde, si nous n'avons pas préalablement trouvé à nos yeux une sorte de cohérence dans notre environnement, si nous n'avons pas d'abord reconstruit ce qui est autour de nous, en situant les choses non seulement entre elles mais également par rapport à nous-mêmes. Le philosophe G. Canguilhem exprime cela au moyen d'une belle métaphore : vivre, c'est rayonner(3). Il veut dire que chacun tentera d'organiser son environnement pour en faire son milieu. Il va l'organiser comme s'il était au centre, en faisant converger vers lui les liens qu'il établit entre les choses, autrement dit en leur accordant de l'importance selon son point de vue à lui. Nous touchons là du doigt ce que nous appellerons un savoir d'expérience : un savoir qui est issu de notre point de vue singulier sur le monde.

En tant que vivant humain, nous sommes profondément ancrés dans le présent de notre vie. Car vivre est un défi de tous les instants puisque les choses ne se font pas toutes seules : rien de ce que nous tentons de faire n'est joué d'avance. Et si nous ne faisons rien, nous sommes emportés par les courants, par les influences; embarqués dans la direction que les contraintes veulent nous faire prendre.

En prenant conscience de cela, nous comprenons que nous sommes des producteurs de sens. Nous ne cessons de vouloir comprendre le monde par rapport à nous-mêmes. Ce n'est pas le 
privilège de quelques-uns, c'est pour chacun un impératif d'existence. Nous sommes immergés dans des milieux contraignants et si nous voulons y vivre, nous devons mobiliser nos énergies, nos équipements biologiques autant que nos facultés intellectuelles - ce qu'Yves Schwartz appelle un corps-soi(1). Cette expérience terre-à-terre, c'est celle du moment présent, quand nos actions enracinées dans le très-actuel génèrent du savoir en continu.

C'est donc la condition humaine qui nous transforme en producteurs de savoir : des êtres en perpétuelle «évaluation », entendue comme une appréciationdu monde qui les entoure, en recherche inquiète d'une prise sur le milieu - une prise au sens très physique, car on s'accroche à la vie dans l'expérience humaine ; mais aussi des êtres en continuel apprentissage car l'épreuve nous enseigne, l'expérience nous fait la leçon.

\section{DEUX APPROCHES COMPLEMENTAIRES DE LA REALITE : SAVOIR ET CONNAITRE}

Une situation se présente et se re-présente à nous, au sens où nous allons la garder en mémoire, en retenir quelque chose. Lorsque nous nous re-présentons, autrement dit lorsque nous nous rendons présent à l'esprit quelque chose que nous ne vivons plus -ou pas encore- dans l'actualité, nous pouvons parler d'un décollement, d'une « désadhérence », en même temps que nous parlons en adhérence de « vivre » comme d'un phénomène à la pointe du moment présent.

Et cela, c'est extraordinaire, prodigieux. L'humain est capable de parler d'une situation passée ou à venir, capable d'énoncer quelque chose à propos de son lien avec le monde, d'exprimer un rapport avec lui en faisant et en se regardant faire : « je sais ce que c'est ; oui, je connais ça! ». Savoir, connaître.... Ce sont des expressions tellement familières que nous perdons de vue ce qu'elles suggèrent. Elles parlent de notre intime expérience du monde, de notre immersion dans la réalité - et simultanément de la leçon retenue en nous dégageant de cette expérience. Une leçon qui nous ouvre la voie de l'anticipation, donc d'une meilleure maîtrise des événements : grâce à elle, nous pouvons nous préparer un peu mieux à ce qui va survenir ; 1'anticiper non pas totalement, mais partiellement.

Le raisonnement sur la condition humaine suppose d'aller au fond de ce que nous éprouvons. Lorsque nous sommes engagés dans une action, nous faisons tous la même expérience. Nous constatons une grande différence entre d'un côté ce que nous pouvons dire de notre activité et de l'autre côté, ce que nous vivons au contact de la réalité. En tant qu'humains, nous avons effectivement deux manières d'entrer en relation avec le monde qui nous entoure. Nous disposons d'abord du langage, du discours, du mode symbolique : cela nous permet une approche indirecte de notre environnement (indirecte puisque nous pouvons en parler à distance, sans y être plongé). Et puis nous avons aussi l'approche directe : c'est le moment présent, celui où nous sommes directement confrontés à la réalité ; le moment où nous n'avons plus forcément besoin des mots parce que le contact est immédiat avec les contraintes environnantes. Lorsque l'ergonome AlainWisner(4)

ISSN 1982-8829 Tempus, actas de saúde colet, Brasília, 13(2), 217-233, jun, 2019. 
analyse les situations productives en distinguant « le travail prescrit » et « le travail réel », c'est exactement en référence à ces deux approches. Celui qui organise le travail se place au plan du discours, forcément réducteur pour mieux analyser, alors que le travailleur lui-même est dans l'action concrète face à une réalité complexe.

Ce double rapport à la réalité est permis par le langage. Nous pouvons grâce à lui à la fois évoluer dans le moment présent et nous en extraire, prendre de la distance. Le résultat fait de nous des producteurs de savoirs, c'est-à-dire les créateurs d'un énoncé sur le monde destiné à être de plus en plus affiné.

Nous allons voir que ce double rapport à la réalité permet de comprendre autrement les termes qui guident ici notre réflexion et de les situer les uns par rapport aux autres : le savoir, la connaissance, la compétence.

Si l'on trace une ligne horizontale, on peut dire que cette question de l'énoncé sur le monde peut se déplacer d'un bout à l'autre. D'un côté, ce sera un énoncé hautement subjectif, chargé d'un point de vue très personnel. De l'autre côté, ce sera un énoncé hautement objectif, détaché des différents points de vue, donc extérieur à tous les partis pris (ce qui est l'objectif de la science).

Pour nommer les deux extrémités de notre ligne, nous utiliserons l'opposition " savoir » et « connaissance ». Mais tout le monde n'est pas d'accord avec cette distribution. C'est un peu une difficulté dans les sciences humaines. Selon les disciplines, il y a des acceptions différentes relevant d'un débat en épistémologie.

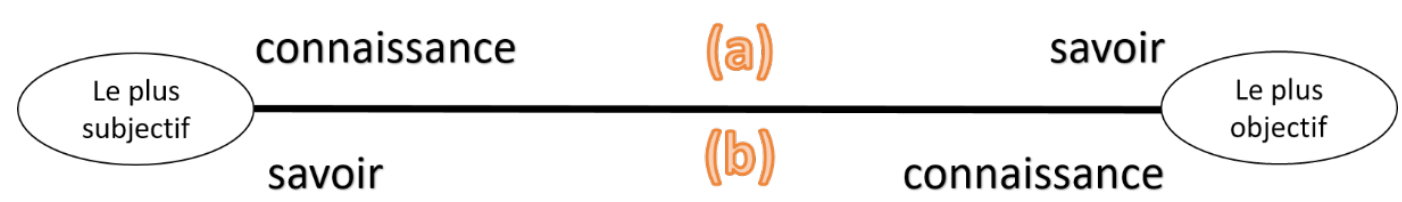

a) "Le savoir " peut ainsi s'entendre comme le contenu objectivé (celui des bibliothèques et banques de données), donc détaché de l'interprétation subjective, détaché des situations singulières et du coup facilement communicable, transmissible. Dans ce cas, c'est « la connaissance » que l'on attribue au sujet. Elle est individuelle, indissociable de la subjectivité, produite par l'expérience personnelle du monde, très liée aux préférences, aux valeurs. On retrouve cette distinction par exemple en pédagogie.

b) Ou bien c'est l'inverse. «Le savoir » se comprend du côté du rapport personnel au monde, du processus d'interprétation par chacun de ce monde (dont il dégage des représentations, des concepts, des croyances). Et dans ce cas, c'est « la connaissance » qui est à l'autre bout, c'est-à-dire comme produit de l'effort d'objectivation, la recherche d'un énoncé dont la vérité est l'objectivité, sans choix en valeur, sans préférence, sans parti pris. Cette distinction est celle de la science et de 
la philosophie - celle que nous adoptons ici.

Entre les deux pôles (le très subjectif / le très objectif), il y a des degrés de prise de distance par rapport au moment de l'action, des degrés de désadhérence. Une organisation par exemple mobilise des savoirs en dehors de la situation présente, mais elle n'est pas neutre, elle a un parti pris : un point de vue de quelque part. Elle est plus éloignée de l'action réelle que ne l'est la procédure ou le mode d'emploi, mais moins éloignée que ne l'est la science. Car celle-ci opère une rupture qui lui permet de se libérer des préférences individuelles, des partis pris. Le scientifique cherche tendanciellement à parler du « point de vue de nulle part »(5).

Qu'appelle-t-on alors un « savoir d'expérience » ? Ce n'est pas un savoir déjà construit, structuré, que l'on trouverait en fouillant dans les herbes touffues de son expérience. Ce sont d'abord des leçons que je retiens de ma rencontre avec certains milieux; c'est donc un savoir très marqué par un point de vue. Cependant cela ne veut pas dire qu'il ne regarde que moi. En effet j'ai vécu quelque chose, une réalité que d'autres n'ont pas forcément vécue et qui peut les intéresser. Un savoir d'expérience est communicable lorsque la personne qui l'a forgé prend un recul critique sur son propre énoncé, sur sa propre interprétation, de manière à distinguer dans le contenu ce qui restera strictement attaché à son point de vue personnel de ce qui, au contraire, dira aux autres quelque chose de nouveau sur une réalité partagée.

On le voit, un savoir d'expérience correspond à un effort de désadhérence tout comme le processus de la connaissance scientifique, mais il ne se confond pas avec lui. Le savoir n'est pas entièrement détaché du point de vue, tandis que la connaissance au contraire pratique l'ablation des points de vue. En voici une illustration. Les savoirs à propos des risques professionnels sont une déclinaison des connaissances scientifiques qui sont partout pertinentes : leur validité est universelle. De leur côté, les savoirs concernant les risques du travail appartiennent aux pratiques des ouvriers de tel ou tel chantier : ils ne sont valables qu'en fonction des spécificités très locales.

Ainsi il est possible de bien différencier le savoir et la connaissance, sans pour autant les rendre incommensurables. La connaissance scientifique vise à circonscrire davantage le monde réel. Précisément pour cette raison, la science est susceptible de s'intéresser au savoir d'expérience, dans la mesure où ce type de savoir permet à la connaissance d'appréhender un aspect de la réalité qui lui est inaccessible directement. Pour connaître tel ou tel aspect de l'expérience humaine, il peut être en effet nécessaire de passer par un point de vue - et non pas rester sur l'approche scientifique qui neutralise le point de vue. Le savoir d'expérience donne ainsi accès à ce qu'aucune approche utilisant les moyens des sciences et des techniques ne pourrait apporter. La science est liée aux seuls faitset elle a besoin des points de vue quand il s'agit de saisir au-delà des faits la question des enjeux, ou encore «l'esprit de la situation ». En ce sens, on peut dire que le savoir d'expérience n'est ni entièrement objectif (car il passe par quelqu'un), ni entièrement subjectif, puisqu'il découvre une partie de la réalité de la situation - certes inaccessible hors de ce point de vue, mais non pas substantiellement dépendant de lui.

ISSN 1982-8829 Tempus, actas de saúde colet, Brasília, 13(2), 217-233, jun, 2019. 
Par exemple, parvenir à vivre le stress dans un service d'hôpital relève d'un savoir d'expérience. Ce n'est pas un « fait » saisissable hors des points de vue des intéressés, c'est-à-dire ici du personnel infirmier. Pourtant, c'est bien une réalité caractéristique de la situation. On voit que la réalité recouvre les faits (eux-mêmes construits par des interprétations critiquées successivement) mais recouvre aussi des points de vue qui disent quelque chose de la réalité comme problème. Chez les ergonomes, l'expression « travail réel »renvoie ainsi à une démarche de connaissance à propos de ce que l'on peut dire de la situation de travail en désadhérence, mais aussi de ce qui s'y vit dans l'adhérence.

Précisons qu'il ne s'agit pas de s'en remettre uniquement aux points de vue. Ce serait verser dans le relativisme, alors qu'il existe bien un réel sur lequel on s'appuie. Cependant nous passons par les points de vue et par la critique de ceux-ci, afin d'accéder à une certaine réalité.

Que signifie enfin la fameuse triade : « savoirs, savoir-faire, savoir-être » ? Cette formuleprétend faire le tour de l'expérience d'une situation. (a) Quelqu'un (b) intervient sur le monde (c) médiatisé par les autres, ses semblables. En d'autres termes : quelqu'un fait quelque chose, donc inévitablement il est en relation avec le monde (ce qui génère un savoir porté par un discours); en relation avec les autres (ce qui génère un savoir-être) et en relation avec lui-même, en tant que corps et personnalité singulière, s'inscrivant dans un espace à un moment donné (ce qui génère un savoir-faire).

L'inconvénient de cette trilogie est de laisser croire que,par les seuls savoirs, on pourrait faire le tour d'une situation vécue. Or ce n'est pas le cas : l'activité déborde les savoirs. Autrement dit, l'activité met en jeu toute la personne humaine de façon largement imprévisible : au plan du discours (les savoirs portés par le langage), au plan qui est en-deçà du discours (« le corpssoi », selon l'expression d'Yves Schwartz) et au plan qui est au-delà du discours : la relation aux semblables humains, les " entités collectives relativement pertinentes " selon le langage d'Yves Schwartz(6). La trilogie reprend effectivement ces trois plans, mais en les traitant tous comme des « savoirs », ce qui revient à vouloir geler l'activitédans la mesure où l'on cherche à l'anticiper en l'enfermant dans des catégories.

\section{L'AGIRET SON JUGEMENT EN TERMES DE COMPETENCE}

La démarche ergologique consiste à tenir ensemble les deux manières d'aborder la réalité dont nous venons de parler. Trop souvent on les confond ou bien on les sépare : c'est à chaque fois une erreur. Confondre le discours sur la réalité et la réalité elle-même, c'est par exemple parler de la compétence comme si c'était une qualité propre à la personne. Or ce n'est pas le cas, puisque nous pouvons être compétent dans telle situation et incompétent dans telle autre. A l'inverse, séparer le discours sur la réalité et la réalité elle-même, c'est par exemple établir une liste de compétences pour un poste et considérer que les titulaires de ce poste peuvent être interchangeables, comme si la personnalité n'avait rien à voir avec le travail à faire.

Tempus, actas de saúde colet, Brasília, 13(2), 217-233, jun, 2019. ISSN 1982-8829 
La démarche ergologique consiste à bien distinguer -sans jamais les séparer- les deux registres d'approche de la réalité :

- D’un côté le discours sur la réalité (dans lequel on retrouvera les plans, les programmes, les référentiels, etc.) ;

- De l'autre côté l'activité réelle : non seulement au sens d'avoir une activité (ce qui est observable), mais aussi au sens d'être en activité (ce qui est largement invisible).

Tandis que G. Canguilhem(7)utilise la distinction traditionnelle en philosophie du concept et de la vie pour différencier ces deux registres, Yves Schwartzparle de désadhérence quand on décolle du moment présent grâce au discours - par contraste avec l'adhérence, au fait de coller à la réalité, d'agir concrètement dans le présent, l'ici-maintenant(8).

En l'illustrant par la question de la compétence, nous venons de dire que c'est une impasse de confondre ou à l'inverse de séparer les deux approches de la réalité. Il convient de les tenir ensemble car chacune de ces approches justifie l'autre. C'est pourquoi, entre le travail prescrit et le travail réel, nous n'allons pas nous contenter d'affirmer qu'il existe un écart. Aussitôt après les avoir distingués, nous reconnaissons qu'ils agissent l'un sur l'autre. L'un existe en fonction de l'autre. Le travail prescrit va cadrer donc anticiper le travail réel, mais le travail réel va déborder le travail prescrit ; il ne sera jamais redondant. Loin d'être une simple application, le travail réel -que le prescrit rend possible en posant des contraintes- va faire surgir un certain nombre d'initiatives. Le prescrit est alors continuellement dépassé par le réel, qui l'entraîne dans une dynamique.

De quelle dynamique parlons-nous ? Du mouvement de la vie et de la pensée : la pensée permet de cadrer l'action et en même temps, c'est l'action donc la vie qui fait bouger le cadre. Ce qui a été pensé à l'avance dans le travail va être continuellement dépassé par tout ce que les travailleurs ont pensé au cours de l'action elle-même. La réciproque ne tarde pas puisque le prescrit va reprendre le dessus, en cherchant à nouveau à anticiper le travail à venir. C'est ce que nous appelons la double anticipation - l'anticipation étant l'initiative que l'on essaie de prendre sur ce qui va arriver pour « faire arriver » quelque chose d'autre. Un programme, un plan de travail, une procédure, un planning, un référentiel, une fiche de poste : ce sont des anticipations. Or ces différentes formalisations du travail (dans le registre des concepts, de la pensée abstraite) vont elles-mêmes agir comme des contraintes qui susciteront indéfiniment des initiatives dans le registre de la vie réelle, la vie concrète. Du côté de la prescription, on a un effort sans cesse renouvelé d'anticiper ce qui va se passer, afin de mieux maîtriser les contraintes que l'on imagine. Du côté de la mise en œuvre, on a également un effort toujours recommencé de prendre des initiatives, de dépasser le prescrit pour dominer les contraintes qui vont réellement surgir. En illustration de cette représentation dynamique, nous pouvons évoquer l'image de la marche : chaque pied anticipe l'autre, avant de se laisser lui-même dépasser.

ISSN 1982-8829 Tempus, actas de saúde colet, Brasília, 13(2), 217-233, jun, 2019. 
Dans le vocabulaire ergologique,nous parlons de « la dialectique des registres » : concept et vie ; adhérence et désadhérence. C'est une manière d'appréhender le travail qui permet de considérer autrement la question de la compétence.

Le couple conceptuel adhérence et désadhérence apporte d'abord un éclairage sur la question de l'acte professionnel. Celui-ci a en effet deux facettes très différentes. Il doit pouvoir être saisi de manière impersonnelle, être anonymisé. Impossible en effet d'organiser le travail ou la formation sans avoir détaché l'acte de la situation spécifique dans laquelle il se manifeste. C'est comme cela que l'on peut répertorier dans un référentiel une série d'actes attendus. Cependant, et c'est le paradoxe, il est tout aussi impossible d'imaginer un acte sans un acteur. Autrement dit l'acte professionnel est inconcevable sans quelqu'un qui va forcément avoir une interprétation très personnelle de ce qui est prescrit. Pour organiser et évaluer le travail, l'identification d'un acte professionnel est par conséquent toujours prise dans une tension entre anonymisation et personnalisation / adhérence et désadhérence.

On reconnaît là un constat très commun que l'on partage lors d'une évaluation, celui d'une sorte de décalage entre le référentiel et la réalité. C'est comme si, en termes de compétences, l'agir d'une personne à un moment donné ne trouvait pas sa traduction fidèle dans le discours. Cela vient de ce que la personne au travail s'inscrit dans un devenir, dans une histoire, dans une continuité, dans la vie avec ses méandres - tandis que le référentiel est un outil conceptuel pour penser l'activité humaine selon une suite logique, pour l'inscrire dans une discontinuité, dans une suite d'actions bien découpées avec un début et une fin clairement repérés. Il ne peut par conséquent y avoir d'homothétie entre le référentiel et l'activité, puisque le premier appartient au registre des concepts (qui suppose la désadhérence, la distance, la discontinuité) tandis que la seconde relève du registre de la vie (qui implique l'adhérence, le très actuel, la continuité). Le référentiel reflète ainsi forcément une vision partielle, approchée, de l'activité. Néanmoins ce référentiel est indispensable, parce qu'on ne peut parler de l'activité sans se référer à une norme. Cette remarque suggère d'ailleurs une certaine manière de faire usage des référentiels, nous allons le voir.

Nous voudrions auparavantrevenir sur ce que peut signifier « agir », car c'est bien l'agir qui est au centre d'une réflexion sur la compétence. Dans la perspective anthropologique présentée ici, agir correspond à une expérience normative. La norme sociale qui structure le travail a besoin de prestige pour être prise au sérieux et être suivie d'effet. Carcontrairement à une loi physique, naturelle, ses effets ne sont pas mécaniques. L'autorité d'une norme sociale viendra de sa position d'antériorité : elle a précédé l'action qui va bientôt se dérouler, donc il faut la prendre au sérieux. On sait aussi que la norme sociale vise l'assujettissement : il s'agit effectivement de lui obéir, d'être conforme. Cependant, comme l'a analysé Canguilhem(7)qui a théorisé la norme, avant Foucault et Bourdieu, la norme a besoin d'un relais d'existence. Autrement dit, la norme représente une contrainte mais elle suppose un être d'initiative qui est capable de la mettre en histoire. Il faut quelqu'un, un être humain avec toute sa singularité, pour prendre au sérieux une règle, une norme, un référentiel - sans quoi cette règle, cette norme, ou ce référentiel n'a aucun avenir.

Tempus, actas de saúde colet, Brasília, 13(2), 217-233, jun, 2019. ISSN 1982-8829 
Nous retrouvons la double anticipation : pour reprendre la métaphore de Wittgenstein(9),agir suppose non pas d'obéir à la norme comme on suit les rails du chemin de fer, mais plutôt de la prendre en compte comme on suit un panneau indicateur. On s'efforce d'aller dans son sens, sans jamais perdre de vue la réalité, le très-actuel. Cela veut dire que nous gardons malgré tout l'initiative. Les indications données en première anticipation, au plan de la désadhérence, sont celles du cadre normatif, dans lequel on peut ranger le programme, le plan de travail, le référentiel, la fiche de poste, etc. De son côté, celui qui doit mettre en œuvre toutes ces prescriptions est placé dans l'adhérence, il est immergé dans un présent dans lequel il maîtrise certaines choses et d'autres non. Son agir ne sera pas une déduction monotone du prescrit, ce sera une prise en compte de ce qui fait contrainte pour lui, en essayant ensuite de reprendre le dessus, d'être plus actif que passif, de reprendre l'initiative, de piloter la situation dans laquelle il se trouve.

Deux remarques ici. D'abord le prescrit n'est pas le seul à faire contrainte en situation; il y a une foule de choses à prendre en compte dans les circonstances du présent de l'action, qui s'ajoutent au prescrit. Ensuite, la contrainte n'est pas forcément négative. Si elle exige toujours d'être prise en compte, elle peut aussi se présenter comme une opportunité pour celui qui agit. C'est toute la nuance entre : «s'adapter », donc accepter une phase d'assujettissement - et puis dans la phase suivante, « adapter », autrement dit tenter d'aménager la contrainte de manière à ce qu'elle serve notre projet d'agir. On voit comment l'image du double dépassement (double anticipation) permet de bien appréhender ces deux phases de l'agir efficace.

\section{UNE APPROCHE CRITIQUE DE LA COMPETENCE}

Ce que nous avons dit jusqu'à présent nous permet de proposer une vision dynamique de la compétence, comme alternative à la vision statique.

La vision statique est celle dénoncée depuis longtemps par des auteurs comme Jean-Pierre Le Goff(10). C'est un certain discours sur la compétence qui paraît déshumanisant parce qu'il décrit la situation de travail sans l'être humain. La compétence se réduit alors à une relation impersonnelle entre l'agir et le savoir. On fait comme si la situation en entreprise n'était qu'un cas de figure informationnel, qu'il suffirait de traiter avec les bons modèles dans les mains des organisateurs. On fait comme si la vie ordinaire au travail était entièrement conditionnée par les cadres normatifs sur lesquels les managers interviennent et orientent l'action.

Dans ce schéma du « vivant simplifié » que dénonçait déjàCanguilhem(11), on crée une rupture entre la vie et le travail. On divise l'agir, donc le sujet humain : les fins d'un côté et les moyens de l'autre. Cela permet au prescripteur de conserver le contrôle de la définition du problème - donc des moyens, des ressources à mobiliser. Et de réduire le travailleur à une « solution », une ressource, un moyen mû par des fins indépendantes de la situation (sa « motivation »).

Les compétences à mobiliser sontainsi définies par l'organisateur du travail comme des 
ressources, amputées par conséquent de l'évaluation - pourtant centrale quand il s'agit de cerner le problème posé. L'acte professionnel est alors considéré indépendamment de l'acteur, puisque la compétence est censée tout dire de l'acte et convoquer ensuite la personne humaineavec ses qualités, sa motivation et ses talents, réduite à exécuter l'acte défini en dehors d'elle et avant elle.

Au contraire, la vision dynamique de la compétence réintroduit le sujet dans son acte. Celui qui travaille n'est pas une sorte de prestataire d'un acte - un acte qui aurait été pensé entièrement par les autres ; il ne se contente pas du rôle d'acteur au travail, il revendique aussi d'être l'auteur de ses actes. Autrement dit, il ambitionne d'être davantage que « normal » c'est-à-dire conforme à l'acte normé : il veut être normatif, capable comme le dit Canguilhem(7) de « faire craquer les normes », de dépasser le prescrit, de le déborder par ses initiatives. Capable de « renormaliser » selon l'expression d'Yves Schwartz(12), ce qui ne veut pas dire que chacun va dicter de nouvelles normes, mais ce qui veut dire qu'il va repenser les conditions de la norme en fonction de soimême et en fonction de l'actualité, du moment de l'action. Dès lors, la norme (ce qui « doit être ", la manière de faire qui est privilégiée par l'organisation) sera repensée, réévaluée dans un icimaintenant. En ce sens on dira que l'acte est réalisé par quelqu'un qui se positionne comme auteur, responsable de cet acte : il a « fait sien » l'acte; il a personnalisé l'acte qui était auparavant formulé de manière anonyme, dans une fiche de poste ou dans un référentiel.

Dans l'approche dynamique, la compétence n'est plus résumée par la liaison « agir-savoirs ». Elle s'inscrit désormais dans un triangle : « agir-valeurs-savoirs ». En d'autres termes, on n'est pas compétent seulement parce qu'on mobilise les savoirs en situation. S'arrêter là, c'est prendre le risque de réduire la compétence à l'apport d'une solution seule, à une simple ressource fournie par quelqu'un d'interchangeable - interchangeable parce qu'il n'aurait pas lui-même nommé le problème.

De là vient sans doute la dérive d'un usage trop mécaniste et déshumanisant de la compétence. Au contraire, que disons-nous lorsque nous introduisons un tiers entre les deux termes, ce tiers qui est appelé ici « les valeurs »? Nous disons que la personne au travailest porteuse et créatrice non seulement de savoirs mais également de valeurs. En effet, placée en situation dans un ici-maintenant, elle va commencer par évaluer, interpréter tout ce qui se passe en gardant pour référence le prescrit [c'est-à-dire ce qu'on lui demande] mais aussi en l'interprétant avec son prisme de valeurs, afin d'apprécier la spécificité du cas qui se présente, de reconnaître les enjeux, de distinguer l'essentiel du secondaire et de hiérarchiser les priorités. La personne qui travaille évalue d'abord - et c'est ensuite qu'elle mobilise les ressources pertinentes de son point de vue.Et en évaluant le moment présent, elle tient compte des autres - car les autres, ses « semblables humains », constituent pour une bonne part la singularité de la situation. Ils sont en effet eux aussi des êtres d'initiative. Cela signifie qu'ils représentent la principale source de variabilité.Chaque initiative prise par un collègue de travail risque de changer la donne, de redessiner la configuration des contraintes à prendre en compte par celui qui est chargé de réaliser la tâche. La première anticipation de la situation, c'està-dire le prescrit, ne pouvait en aucun cas cerner toute cette histoire locale, largement inédite.

Tempus, actas de saúde colet, Brasília, 13(2), 217-233, jun, 2019. ISSN 1982-8829 
Le prescrit correspond à l'initiative de la contrainte : cependant la liste n'est jamais exhaustive puisque de nouvelles contraintes dans l'action ne cessent de resurgir à mesure que l'on répond aux premières. Le protagoniste d'une situation de travail est toujours placé en deuxième anticipation : il lui faut reprendre l'initiative dans la contrainte, à l'intérieur de celle-ci.

La perspective ergologique est davantage attentive aux contradictions de la vie réelle et cela lui permet de défendre une approche dynamique de la compétence. Cette perspective ne s'arrête pas à la situation connue (désadhérence) et essaie de prendre également en compte la situation vécue (adhérence). Cherchant ainsi à s'extraire d'une vision à la fois dualiste et solipsiste de l'expérience humaine, elle est centrée sur une dynamique «Homme-Milieu » telle qu'héritée des travaux de Canguilhem. C'est le mouvement dialectique qui définit ici l'activité.

Lorsque j'entame l'action, je ne maîtrise pas tout. L'effort de connaître, c'est-à-dire l'effort de désadhérence, est certes essentiel pour la réussite car il vise à anticiper ce qui peut l'être. Toutefois il est impossible de prétendre tout maîtriser, tout rationaliser, tout standardiser pour être à l'abri des imprévus au moment d'intervenir. Si l'effort de connaître permet de créer les conditions du travail, il est simultanément accompagné d'un effort de vivre (en adhérence), autrement ditune lutte toujours renouvelée avec les contraintes non anticipées qui ne cessent de surgir aussitôt que j'agis. J'interviens face au réel qui me résiste, dans un monde médiatisé par les autres. C'est une lutte de tous les instants pour y arriver malgré tout et tenter de reprendre l'initiative. C'est cela qui nous renvoie constamment à l'adhérence, dans la relation Homme-Milieu. Agir avec compétence, dans notre perspective, c'est non seulement mobiliser les savoirs requis par la situation -comme l'affirme avec raison Guy Le Boterf(13) - mais c'est aussi être guidé par un point de vue sur la cohérence de cette situation où « tout se mélange ». Ce point de vue, que nul ne peut prétendre avoir à la place d'un autre, résulte du croisement in situ : " effort de vivre / effort de connaître ». Ce point de vue oriente les arbitrages décisifs dans l'action, ces arbitrages qui orientent les faits, les déplacent et font que l'histoire s'écrit au jour le jour, y compris dans le plus micro de nos actes. La compétence dans une perspective dynamique, c'est la maîtrise de savoirs pour agir, mais avec un point de vue au sens fort, un point de vue qui permet de gérer la situation en termes de valeurs, et pas uniquement en termes de savoirs.

\section{QUE FAIRE AVEC LA COMPETENCE DYNAMIQUE?}

Nous avons vu qu'une représentation dynamique de la compétence réintroduit le sujet dans son acte : la subjectivité est prise en compte dans l'action normée au travail. Et en même temps, elle n'empêche pas la nécessaire gestion anonyme, impersonnelle, de cette compétence. Cette gestion est indispensable pour organiser et rendre possible le travail et la formation.

La dynamique de la compétence se comprend avec le schéma de la double anticipation, celui qui permet de tenir ensemble l'impératif de la dépersonnalisation et celui de la personnalisation de l'acte professionnel. Ainsi le travail est à la fois en rupture avec la vie ordinaire et en continuité

ISSN 1982-8829 Tempus, actas de saúde colet, Brasília, 13(2), 217-233, jun, 2019. 
avec elle. En rupture, parce que les actes sont pré-pensés, normés, en première anticipation, ce qui n'est pas le cas dans la vie quotidienne. Et en continuité parce que c'est toujours un être humain, être d'évaluation et d'initiative, qui prend le relais en deuxième anticipation. Pour cela, il faut à la fois : (a) distinguer l'acte et l'acteur, afin de pouvoir gérer l'acte lui-même dans une organisation, sans référence explicite à l'acteur et (b) les tenir ensemble, donc ne pas séparer l'acte et l'acteur, maintenir ce qui les relie - à savoir la subjectivité. Ainsi on se donnera les moyens de rendre compte à la fois (1) de la logique d'une organisation du travail, qui distribue les tâches en définissant les différents actes professionnels à réaliser et les compétences requises - et (2) de la cohérence axiologique et existentielle qui constitue l'expérience du travailleur et le pivot de sa compétence.

Pour tenir ensemble ces deux exigences paradoxales, Yves Schwartz(14) proposait, dès le début des années 1990 à Aix-en-Provence, le modèle des « six ingrédients de la compétence».On notera qu'il est question d'ingrédients plutôt que de critères, afin de souligner les contours estompés de chacun d'eux.

Les trois premiers ingrédients renvoient à l'acte, tandis que les deux derniers renvoient à l'acteur. L'acte peut être évalué en tant qu'il est observable, manifestant une intelligence de la situation. Mais il est lui-même précédé par deux sources de savoirs à mobiliser, les savoirs codifiés plus ou moins maîtrisés d'une part et d'autre part les savoirs liés à la spécificité de la situation, y compris incorporés, car le prescrit doit être actualisé. Ces trois ingrédients de base sont complétés par les trois autres. Ceux-ci correspondent à l'acteur, à son positionnement (son interprétation, son point de vue qui lui permet de gérer le débat sur les manières de s'y prendre, inhérent à toute situation) et à ce qui en découle, c'est-à-dire la mobilisation relative de ses ressources propres. Ce sontlà deux ingrédients -les quatrième et cinquième de la liste- qui sont complétés par un sixième : l'ouverture aux autres personnes impliquées dans la situation de travail. Il s'agit de la dimension collective de la compétence.

La force de ce schéma proposé par Y. Schwartz est de permettre de reconnaître en même temps la facette de la compétence qui est utile à sa gestion (il est possible de l'indexer jusqu'à un certain point, avec les trois premiers ingrédients) et la facette profondément singulière -impossible à référencer- de cette même compétence, identifiable avec les trois derniers ingrédients. Il faut souligner que les deux sous-parties de la liste [l'acte- l'acteur] sont bien reliées et non simplement juxtaposées, puisque Y. Schwartz précise d'emblée que les ingrédients 4, 5 et 6 , ceux de l'acteur, ont un effet récurrent sur l'acte lui-même $(1,2,3)$, en positif ou en négatif. L'acte professionnel tel qu'il est défini par l'organisation sera nécessairement réinvesti par l'acteur, ce qui explique qu'il soit toujours profondément singulier, alors même qu'on peut le reconnaître dans sa généralité selon l'angle adopté : avec un point de vue éloigné ou au contraire rapproché.

Les réflexions qui précèdent incitent à de nouvelles pratiques. Comment par exemple utiliser un référentiel lorsque nous adoptons une vision dynamique de la compétence ? Dans un premier temps, l'évaluation porte sur l'exercice singulier d'une personne à qui l'on a confié une tâche. 
Cette personne défend une interprétation de la situation qui est soumise à débat, c'est-à-dire à un croisement de points de vue. Le référentiel va par la suite offrir une pierre de touche, et donc en quelque sorte " un garde-fou » pour se protéger de l'arbitraire des jugements de valeurs. On constate une série de faits, que l'on recoupe avec d'autres faits, ceux indexés dans le référentiel, pour en conclure par inférence du réel au possible, autrement dit se prononcer surla capacité de telle personne à réaliser de tels actes professionnels à l'avenir. Il existe toujours une marge de débat, que l'on pondère avec l'évaluation - celle-ci relevant également d'une sorte de pari interprétatif. Bref, on utilise la stabilité d'un référentiel (par ailleurs, lui-même issu d'un débat) pour donner à l'évaluation à la fois une pertinence et une légitimité.

Ajoutons que l'on gagnerait à distinguer le singulier et le pluriel du mot compétence. La compétence au singulier nous paraît être une appréciation générale qui correspond à une approche globale de celui qui agit : elle sanctionne et encourage la prise de distance de l'acteur, ainsi que la formation de son point de vue. Le débat interprétatif sert à comprendre le rapport aux normes, celui qui amène la personne à dépasser le prescrit, en réinterprétant sa tâche dans le service. De leur côté, les items du référentiel viennent appuyer cet effort d'interprétation en se fondant sur des faits, des actes ; en proposant la formulation « être capable de » qui identifie des compétences au pluriel, anonymisées, comme un essai de généralisation avec des repères objectifs et une volonté de s'extraire de l'immédiateté, de la particularité de la tâche.

\section{CONCLUSION}

Nous avons adopté la perspective anthropologique de G. Canguilhem, reprise par Y Schwartz, pour réinterpréter quelques-uns des termes appartenant au champ de l'approche par compétence, toujours plus présente aujourd'hui dans les questions d'organisation du travail et de la formation. Dans cette perspective, l'être humain est à la fois profondément social et profondément singulier. Parce qu'il est social, ses actes seront largement codifiés : définis à l'avance dans toutes sortes de référentiels, de cadres normatifs, que ce soit pour l'apprentissage ou pour la vie professionnelle. Parce qu'il est singulier, ses mêmes actes seront chaque fois investis par une histoire, un corps propre, une personnalité. C'est pourquoi l'activité humaine se comprend comme une dialectique entre norme et renormalisation, entre désadhérence et adhérence. Considéré en formation ou au travail, l'acte individuel est regardé comme impersonnel sous l'angle de sa gestion - mais personnel sous l'angle de l'activité. Si l'on ne tient pas ensemble ces deux facettes de l'acte, on prend le risque d'un mésusage du terme de compétence, puisque celle-ci doit permettre d'apprécier l'agir professionnel. Le premier écueil sera de ne considérer que les actes seuls dans l'évaluation, sans s'inquiéter de l'acteur, de son point de vue, de ses intentions. Le second écueil sera de ne regarder que l'acteur seul, sans s'inquiéter de ses actes, de ce qu'il a dû affronter comme réalité en accomplissant sa tâche. Il faut en réalité passer par l'acte, mais sans s'y arrêter car il s'agit de parler de l'acteur en termes de compétence.

Formulé dans les années 1990 en France (15), le projet d'évoluer progressivement de la 
qualification vers la compétence pour parler du travail et de la formation, consistait àse rapprocher de la réalité des situations de plus en plus changeantes dans la vie réelle. D'une prise de distance claire et affichée, celle de la qualification, on est passéà une tentative d'adhérence à la situation, avec la compétence et sa proximité au travail réel. Toutefois, cette proximité ne saurait être totale. En effet, s'il s'agissait uniquement d'apprécier ce qui est fait ponctuellement, l'évaluation serait de l'ordre du constat immédiat, avec une validité très réduite, sans aucun pronostic, sans aucune hypothèse sur la maîtrise des situations à venir. La notion de compétence doit intégrer un minimum de désadhérence, de décollement par rapport à la situation rencontrée. En reconnaissant qu'une personne est compétente, nous disons qu'elle a apporté les preuves par l'acte, mais qu'elle peut aller au-delà, plus tard et encore ailleurs, pour réussir des actes similaires dans une même famille de situations.

Toutefois ce n'est pas l'acte qui est transféré d'un environnement à un autre, c'est l'acteur qui opère ce transfert : d'où l'intérêt de la notion de « compétence transversale ", pour indiquer les dispositions acquises par l'acteur afin non seulement de transférer dans une même famille de situations, mais également de passer d'une famille de situations à une autre.

Finalement, à partir d'un éclairage anthropologique sur le travail et l'apprentissage, nous pouvons regarder la compétence dans une perspective dynamique. La compétence, c'est la maîtrise de savoirs au moment d'agir en situation : cela permet sa codification, son indexation dans des référentiels, sa reconnaissance sociale. Mais cette maîtrise des savoirs s'accompagne d'un point de vue fort qui permet à chaque fois de gérer la situation singulièrement, en mobilisant les savoirs en fonction d'une évaluation très personnelle, d'un positionnement en valeurs. La compétence entendue comme une dynamique est suffisamment identifiable pour être référencée et suffisamment ouverte à l'interprétation pour être constamment renouvelée en situation.

\section{RÉFÉRENCES}

1. Schwartz Y. Le paradigme ergologique ou un métier de philosophe. Toulouse, France: Octares; 2000.

2. Canguilhem G, Zaloszyc A. Écrits sur la médecine. Paris, France: Éditions du Seuil; 2002. p. 68

3. Canguilhem G. La connaissance de la vie. Paris, France: Librairie philosophique J. Vrin; 1998. p. 147

4. Wisner A. Réflexions sur l'ergonomie: 1962-1995. Toulouse, France: Octares éditions; 1995.

5. Nagel T. Le point de vue de nulle part. Combas, France: Éd. de l’Éclat; 1993. 
6. Schwartz, Yves. De l'inconfort intellectuel : ou comment penser les activités humaines ? In: Cours-Salies P, éditeur. La liberté du travail. Paris, France: Editions Syllepse; 1995. p. 117.

7. Canguilhem G. Le normal et le pathologique. Paris, France: Presses universitaires de France; 1999. p. 179

8. Schwartz Yves. Produire des savoirs entre adhérence et désadhérence. In : Cerf, M. Béguin, P, éditeurs. Dynamique des savoirs, dynamique des changements. Toulouse: Octarès; 2009.p. 15-28

9. Laugier S, Chauviré C, éditeurs. Lire les « Recherches philosophiques ». Paris, France: J. Vrin; 2006. p. 131-156

10. Le Goff J-P. La barbarie douce: la modernisation aveugle des entreprises et de l'école. Paris, France: La Découverte; 1999. p. 34

11. Canguilhem, Georges. Milieu et Normes de l'Homme au Travail. Cahiers Internationaux de Sociologie. 1947. (3). p. 120-136.

12. Schwartz Y, Durrive L. Entretiens sur l'activité humaine. Toulouse: Octarès; 2003.

13. Le Boterf G. Repenser la compétence: pour dépasser les idées reçues : quinze propositions. Paris, France: Eyrolles-Éd. d'organisation; 2008.

14. Schwartz, Yves. Les ingrédients de la compétence : un exercice nécessaire pour une question insoluble. Educ Perm. 1997;(133).

15. Zarifian P. Objectif compétence. Rueil-Malmaison, France: Éditions Liaisons; 1999.

Artigo apresentado em: janeiro 2019

Artigo aprovado em: março 2019

Conflito de interesses: o autor declara não haver conflito de interesses

Suporte financeiro: não 